\title{
Accurate iterative analytic solution of the Kapchinskij-Vladimirskij equations for the case of a matched beam
}

\author{
Oscar A. Anderson \\ Lawrence Berkeley National Laboratory \\ University of California, Berkeley, \\ Californic 94720, USA
}

(Dated: October 1, 2006)

\begin{abstract}
The well-known Kapchinslfj-Vladimirskij (KV) equatione are difficult to solve in general, but the problem is simplified for the matched-beam case with sufficient symmetry. We show that the interdependence of the two KV equations is eliminated, so that only one needs to be solved-a great simplification. We present an iterative method of solution which can potentially yield any desired level of accuracy. The lowest level, the well-known smooth approximation, yielde simple, explicit results with good accuracy for weal or moderate focusing fields. The next level improves the accuracy for high fielda; we previously showed [Part. Accel. 52, 133 (1996)] how to maintain a simple explicit format for the results. That paper used expansion in a amall parameter to obtain the second level. The present paper, using straightforward iteration, obtains equations of first, second, and third levels of accuracy. For a periodic lattice with beam matched to lattice, we use the lattice and beam parameters as input and solve for phase advances and envelope waveforms. We find excellent agreement with numerical solutions over a wide range of beam emitiances and intensities.
\end{abstract}

\section{INTRODUCTION}

This paper analyzes matched beams in alternatinggradient (A-G) systems. Such beams have smaller excursions than mismatched beams, thus requiring smaller transport apertures, and are the starting point for analysis of mismatched beams. Designers and experimenters who work with A-G systems need simple, accurate ways to predict the performance of matched beams. One usually begins with the coupled Kapchinskij-Vladimirskij (KV) equations [1]. For weak focusing fields, solution by the smooth approximation [2], [3], [4] is reasonably accurate. Methods of solution for strong fields [5], [6], [7] have tended to be indirect or complex. Lee, using a double expansion in focus strength and emittance, demonstrated high accuracy for a special case [8]. Our previous paper [9] presented general results in a simple explicit format, but with only moderate accuracy.

The present paper, which was summarized in Ref. [10], uses a convenient iteration method to obtain explicit results. High accuracy is demonstrated for a wide range of parameters, which are: the A-G field strength; beam emittance; and beam charge or current. These would be small parameters if normalized (cf. App. J), but we use physical urits throughout. We assume the latitice to be periodic with the beam matched to the lattice, i.e., having the same periodicity. The lattice is assumed symmetric. (Asymmetric cases are treated with a novel numerical technique by Lund et al. in Ref. [11].)

We solve explicitly for average radius [Eq. (30)], peak radius [Eq. (36)], and the phase advances [Eqs. (40) and (43)]. We give these results for three levels of accuracy and complexity. All these formulas apply to arbitrary symmetric lattices. They require at most the 3rd and 5th harmonics of the lattice profile, becoming simpler for smooth profiles (App. G). Envelope waveforms are given in Appendix E and illustrated in Fig. 2.
Summary: We begin with the coupled KV equations and show in Sec. III that the matching assumption decouples them so that only one equation needs to be solved. (We are not aware that this simplification has been previously noted.) Section IV expands the $a(z)$ envelope about its mean and then splits the resulting equation into its average part $A$ and periodic part $\rho$. The differential equation for $\rho(z)$ is solved (Sec. V) by iteration. These results are combined to obtain a matching equation for the average radius $A$. This equation is written to various orders of accuracy; our special definition of "order" is given in Sec. V. The results (Sec. VI) are compared to the results of numerical solution of the full KV equations. The first-order case is tisually called the smooth approximation. Second- and third-order terms increasingly improve the accuracy. Over a wide range of parameters, ow third-order versions provide a great. improvement over those previously published [2]-[6]. .

Section VII combires results from Secs. V and VI to give the maximum and minimum radii. The phase advances $\sigma$ and $\sigma_{0}$ are given in Sec. VII. There is a useful approximation for $\sigma_{0}$ in Sec. IX. Appendices F, G, and H discuss the use of Fourier analysis of the A-G focusing waveform to facilitate the solution. Some exact formulas for the FODO case are given in Appendix $I$.

\section{THE KV EQUATIOIS AND SYMMMETRIC LATTICE MODEL}

The KV equations for envelopes $a(z)$ and $b(z)$ are [1]

$$
\begin{aligned}
& a(z)^{\prime \prime}=-K(z) a+\frac{\xi^{2}}{a^{3}}+\frac{2 Q}{a+b} \\
& b(z)^{\prime \prime}=+K(z) b+\frac{\xi^{2}}{b^{3}}+\frac{2 Q}{a+b}
\end{aligned}
$$


with normalized beam current $Q$, emittance $E$, and lattice focus-strength function $K(z)$. The latter is periodic over the lattice cell length, defined as $2 L$, so that $K(z+2 L)=K(z)$. In practice, the lattice cell has one or two symmetries. The double symmetry occurs if the focus drift spaces are equal in length. In this paper we assume that the function $K(z)$ has odd symmetry at the center of the drift spaces and even symmetry at midelectrode points. These symmetry points necessarily alternate at intervals $L / 2$. It is convenient in what follows to choose one of the even points for the $z$ origin. (The odd choice is appropriate for the case of unequal drift lengths; that case is not discussed in the present paper.) Then the double symmetry is expressed as

$$
\begin{aligned}
K(L-z) & =K(L+z), \\
K(L / 2-z) & =-K(L / 2+z) .
\end{aligned}
$$

It follows that $K(z)$ is anti-symmetric about $3 L / 2$ and that $K(z)$ obeys the shift relations

$$
\begin{aligned}
K(z+L) & =-K(z), \\
K(z+2 L) & =+K(z) .
\end{aligned}
$$

It also follows that

$$
K(-z)=K(z)
$$

Equation (3d) confirms the lattice periodicity which was assumed above. Equations $(3 a)$ and ( $3 b$ ) indicate that $K(z)$ can be expanded as a cosine series containing only odd-numbered harmonics.

In this papar we solve Eqs. (1) and (2) for the beam envelopes in the special case where the bearn is matched, i.e., $a(z)$ and $b(z)$ have the same periodicity as the lattice. The initial conditions with the symmetry of Eq. (3e) are $a^{\prime}(0)=0, b^{\prime}(0)=0, a(0)=a_{0}, b(0)=b_{0}$.

For given $Q$ and $E$ and given latitice parameters, $a_{0}$ and $b_{0}$ can be chosen over wide rauges but the bearn will be matched only for particular values, which we call $a_{\mathrm{m}}$ and $b_{\mathrm{m}}$, such that $a(2 L)=a_{\mathrm{m}}$ and $b(2 L)=b_{\mathrm{m}}$. In this cese, the initial conditions are

$$
a^{\prime}(0)=0, b^{\prime}(0)=0, a(0)=a_{\mathrm{m}}, b(0)=b_{\mathrm{m}}
$$

If we substitute $a_{L}(z) \equiv a(z+L)$ and $b_{L}(z) \equiv b(z+L)$ in Eqs. (1) and (2) and use Eq. (3c), we find that $a_{L}(z)$ and $b_{L}(z)$ also satisfy the KV equations with transposed initial conditions: $a_{L}(0)=b_{\mathrm{m}}, b_{L}(0)=a_{\mathrm{m}}$.

We deduce that for a matched beam $b(z)=a(z+L)$, so that the denominator of the last term in Eq. (1) can be written $a(z)+b(z)=a(z)+a(z+L)$. Then $b(z)$ does not appear and Eq. (1) is decoupled. In Sec. III and App. A we find an expression for the function $[a(z)+a(z+L)]^{-1}$ in terms of integrals involving the given lattice function $\Gamma(z)$. After that, Eq. (2) is not needed for our matchedbeam case. The main work of this paper will be to find $a_{\mathrm{m}}$ and $b_{\mathrm{m}} \rightarrow$ see Secs. IV through VII. First we introduce some definitions.

\section{Operators, Functions, Paraneters, Derived Quantities}

To aid the solution of Eqs. (1) and (2), we define in Table I the operators $\{\ldots\rangle,\{\ldots\}, \int, \iint$

\begin{tabular}{|c|c|c|}
\hline & $\langle f\rangle \equiv(1 / 2 L) \int_{0}^{2 L} f(z) d z_{1}$ & (4) \\
\hline & $\{f\} \equiv f-\langle f\rangle$. & (5) \\
\hline For even $\psi(z) \exists\langle\psi\rangle=0$ & $\int \psi \equiv \int_{0}^{z} \psi\left(z^{\prime}\right) d z^{\prime}$ & (6) \\
\hline and & $\iint \psi \equiv\left\{\int_{0}^{z} d z^{\prime} \int_{0}^{z} \psi\left(z^{\prime \prime}\right) d z^{\prime \prime}\right\}$. & (7) \\
\hline & $k \equiv K(0)_{+}$ & (8) \\
\hline & $h(z) \equiv K(z) / k_{1}$ & (9) \\
\hline & $g \equiv \iint h$ & (10) \\
\hline & $\delta(z) \equiv \iint\{h g\}$, & (11) \\
\hline & $A \equiv\langle a(z)\rangle$ & (12) \\
\hline & $\rho(z) \equiv(a(z)-A) / A_{1}$ & (13) \\
\hline & $\alpha \equiv 3 \in^{2} / A^{4}$ & (14) \\
\hline & $\beta \equiv \alpha L^{3} / \pi^{2}$, & (15) \\
\hline & $q \equiv Q / A^{2}$, & (16) \\
\hline & $K^{\mathrm{cff}} \equiv K^{2}\left\langle\left[\int h\right]^{2}\right\rangle$ & $(1 \bar{T})$ \\
\hline & $\Phi \equiv 3 k^{2}\left\langle g^{2}\right\rangle$ & (18) \\
\hline & $\rho_{\mathrm{m}} \equiv h_{1} / L^{2} / \pi^{7}$ & (19) \\
\hline
\end{tabular}
which operate on even, periodic functions; the functions $h(z), g(z), \delta(z), \cdot \rho(z) ;$ and the constants $k, \alpha_{1} \beta, q, A, K^{\mathrm{eff}}, \Phi$, and $\rho_{\mathrm{m}}$. In Eq. (19), $h_{1}$ is the first Fourier coeficient of $h(z)$-ct. App. F.

TABLE I: Collection of definitions to be used in this paper

In Table $I$, the operator $\langle\ldots\rangle$ performs an average over a cell length $2 L$. The operator $\{\ldots\}$ removes the average part of a periodic function: e.g., $2\left\{\cos ^{2} x\right\}=$ $\{1+\cos 2 x\}=\cos 2 x$. The operator $\iint$ operates on even periodic functions that have zero average. It gives the repeated indefinite integral and removes the average part, if any, of the result. This removal can be implemented by constructing a suitable lower limit for the outer integral. To illustrate, Eq. (10) can be written

$$
g \equiv \int_{L / 2}^{z} d z^{\prime} \int_{0}^{z^{\prime}} h\left(z^{\prime \prime}\right) d z^{\prime \prime},
$$

which subtracts the value at $L / 2$, so that $g(L / 2)=0$. Alternatively, one can start both integrals at zero and then apply the operator $\{\ldots\}$, as in Eq. (7). For example, $\iint \cos z=\{\sin z\}=\{1-\cos z\}=-\cos z$. Note that, from Eq. (9), $h(0)=1$. 


\section{DECOUPLING THE KV EQUATIONS FOR SYMMETRIC MATCHED-BEAM INITIAL GONDITIONS}

For a matched beam with the symmetries of $\mathrm{Eq}$. (3), we showed in Sec. II that $b(z)=a(z+L)$, implying that Eqs. (1) and (2) are decoupled and that $\langle a\rangle=\langle b\rangle \equiv A$. We write

$$
a(z) \equiv A(1+\rho(z)), \quad b(z) \equiv A(1+\rho(z+L)),
$$

with $\rho(z)>-1$ for all $z$. Then the $Q$ term in $\mathrm{Eq}$. (I) can be written

$$
\frac{2 Q}{a+b}=\frac{Q}{A}(I-F(h(z)))
$$

where $F$ is obtained from an expansion and iterations. All terms consist of integrations of the focus-strength profile $h(z)$ [Appendix A]:

$$
F(h(z))=k^{2} \iint\left\{h \iint h\right\}+\cdots \equiv \delta(z)+\cdots .
$$

Additional terms are shown in the appendix. With the lattice symmetries of Eq. (3), all the series terms for $F(z)$, including $\delta(z)$, have only even-numbered cosine harmonics-in contrast to $h(z)$, which has only odd ones.

Equetions (1) and (2) are decoupled to all orders so that Eq. (2) is superfluous from here on. Equations (1) and (2) are replaced by

$$
\begin{gathered}
a(z)^{\prime \prime}=-K(z) a+\frac{E^{2}}{a^{3}}+\frac{Q}{A}\left(1-k^{2} \delta(z)+\cdots\right), \\
b(z)=a(z+L) .
\end{gathered}
$$

\section{EXPANDING AND DECOMPOSING INTO AVERAGE AND PERIODIC PARTS}

Substituting $a=A(1+p(z))$ in the first three terms of Eq. (1), expanding $1 / a^{3}$, dividing by $A$, and using (22) and (14), Eq. (1*) is equivalent to

$$
\begin{aligned}
& \rho(z)^{\prime \prime}=-k h(z)-k h(z) \rho+ \\
& \quad \frac{\alpha}{3}\left(1-3 \rho+6 p^{2}-10 \rho^{3}+15 \rho^{4} \cdots\right)+q\left(1-k^{2} \delta(z)+\cdots\right) .
\end{aligned}
$$

To solve for the ripple $\rho(z)$ and for the mean radius $A$ (which appears in the definition of $\alpha$ and $q$ ), we decompose Eq. (23) into a pair of equations. Averaging Eq. (23),

$0=-k\langle h \rho\rangle+\frac{\alpha}{3}+2 \alpha\left\langle\rho^{2}\right\rangle-\frac{10}{3} \alpha\left\langle\rho^{3}\right\rangle+5 \alpha\left\langle\rho^{4}\right\rangle+\cdots+q$.

Subtracting Eq. (24) from (23),

$$
\begin{aligned}
\rho^{\prime \prime}= & -h h(z)-h\{h \rho\}-\alpha \rho+2 \alpha\left\{\rho^{2}\right\} \\
& -\frac{10}{3} \alpha\left\{\rho^{3}\right\}+5 \alpha\left\{\rho^{4}\right\}+\cdots-q k^{2} \delta(z)+\cdots,
\end{aligned}
$$

with the $\{\cdots\}$ operator defined by Eq. (5). There are now two equations, each containing $\rho(z)$ and $A$ - the lat ter represented by $\alpha$. These represent the KV equations (1) and (2) for our matched beam. We will obtain $A$ and $\rho(z)$ to good accuracy in Secs. TV-VI and App. E.

\section{ITERATIVE SOLUTION: ARBITRARY SYMMETRIC FOCUSING PROFIIE}

On the right-hand side of Eq. (25), the $k h(z)$ term dominates the terms involving the unlnown function $\rho(z)$. Therefore, we use only $k h(z)$ in the initial integrations which give $\rho_{(0)}$. Then we insert $\rho_{(0)}$ into (25) and integrate again to obtain $\rho_{(1)}$. (A miniseule term, $q k^{2} \delta(z)$, in $\rho(0)$ is omitted.) This process is repeated to get $\rho(2)$ :

$$
\begin{aligned}
& \rho_{(0)}=-k g, \\
& \rho_{(1)}=\rho_{(0)}+\alpha k \iint g+k^{2} \delta+\frac{10}{3} \alpha k^{3} \iint g^{3}, \\
& \rho_{(2)}=\rho_{(1)}-\alpha^{2} k \iiint \int g-k^{3} \iint h \delta-4 \alpha k^{3} \iint g \delta .
\end{aligned}
$$

In (26c), $\iiint \int$ stands for two applications of the operator $\iint$ defined by $\mathrm{Eq}$. (7).

To complete the approximate solution of the $\mathrm{KV}$ equations, $\rho(z)$ from $\mathrm{Eq}$. (26) is put into the matching equation (24). In Eq. (26) we included no items (e.g., $\left.2 \alpha k^{2} \iint\{g\}^{2}\right)$ that would give terms in (24) higher than third power in the parameters $k^{2}, \alpha$, and $q$. That is, we go no higher than third order as defined below.

Some tarms venish by orthogonality, since $h(z), g(z)$, $g^{3}$, etc., possess only odd harmonics while $\delta(z),\left\{g^{2}\right\}$, $\left\{g^{4}\right\}$ have only even ones for symmetric quadrupoles.

A term not shown, $q k^{2} \iint \delta(z)$, involves multiple integrations of an already small function and would contribute $<0.04 \%$ to the maximum radius $a_{\max }$ even at $\sigma_{0}=120^{\circ}$ and affect $A$ by less than 2 parts in 10,000. This is much smaller than the two parts per thousand critarion used for significant terms in Appendix $\mathrm{E}$.

Order: We reckon the order of a term in the matching equation by counting the number of factors $k^{2}, \alpha$, and $g$. These would become small parameters in a nondimensional formalism (Appendix J). We prefer to retain physical units for quantities such as the axial coordinate $z$. Note that Lee [8] defines order using $k$ and $E$ so that our third order corresponds approximately to his fifth order. Appendix $B$ evaluates the combination of Eqs. (24) and (26). The result, in simplified form, is shown in the next section.

\section{MATCEING EQUATION TO VARIOUS ORDERS FOR AN ARBITRARY SYMMETRIC LATTICE}

Third Order: Inserting Eq. (26) into Eq. (24) yields seven terms [App. B, Eq. (B7)]. Some terms combine, 

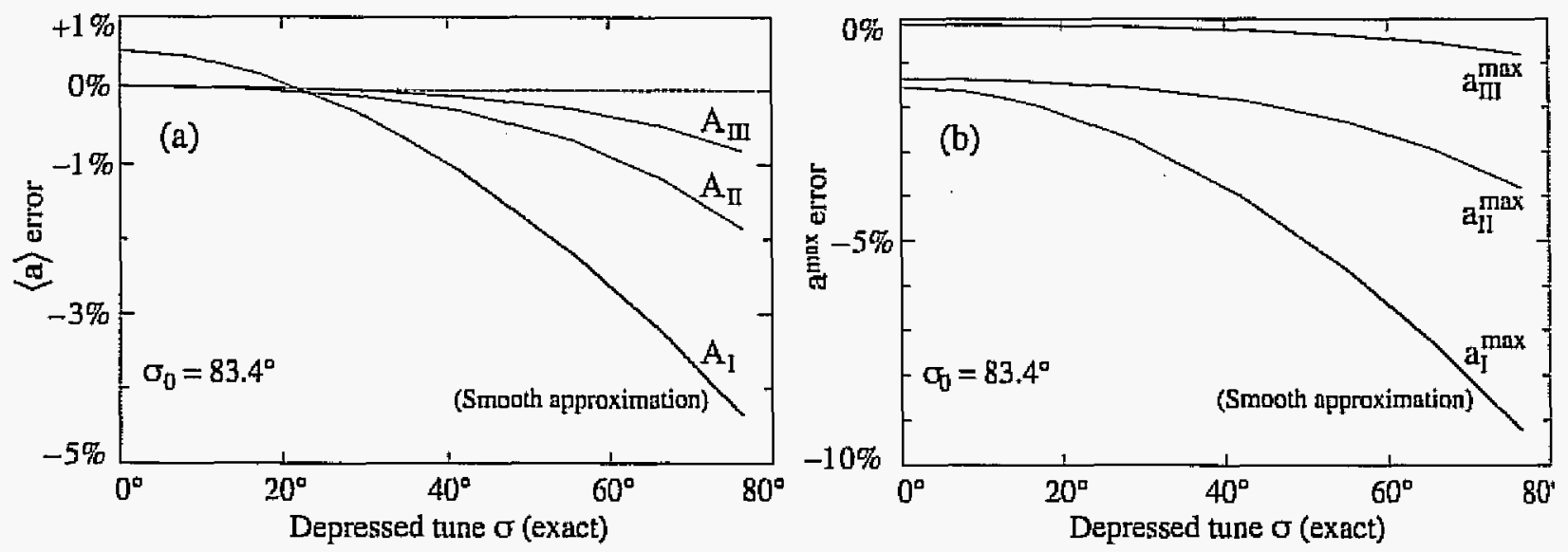

FIG. 1: Пlustration of results for epecial case of FODO lattice, compared with simulation results. (a) Accuracy of mean radius, from Eqs. (30), (32), (33). (b) Accuracy of maximum radius, from Eqs. (36), (37), (38).

Input quantities; $Q, E$, and quad voltage $V_{Q}(\propto K)$. Other parameters: aee Table II. $V_{Q}$, fixed at $20 \mathrm{kV}$, gives phage advance $\sigma_{0}$ of $83.37^{\circ} ; E$ and $Q$ are varied so that depressed tune $\sigma$ ranges between $0^{\circ}$ and $76.5^{\circ}$; exact $\sigma_{0}$ and $\sigma$ are obtained numerically.

TABLE II: Parameters used in Fig5. 1a and 1b, based on the actual MFE ESQ accelerator [12].

\begin{tabular}{llll}
\hline \hline Quad cell length & $20 \mathrm{~cm}$ & Quad voltage (Figs. 1, 3a) & $20 \mathrm{kV}$ \\
Occupancy factor & 0.5 & Quad voltage (Fig. 2) & $25 \mathrm{kV}$ \\
Quad radius ( $\left.a_{Q}\right)$ & $1.75 \mathrm{~cm}$ & Beam current (Fig. 2) & $0.5 \mathrm{Amp}$ \\
Beam entergy & $200 \mathrm{KeV}$ & Norm ernittance (Fig. 2) & $1.65 \pi \mathrm{mrad}-\mathrm{cm}$ \\
Beam particles & $\mathrm{H}^{-}$ions & & \\
\hline \hline
\end{tabular}

resulting in (Appendix C)

where

$$
K_{\dagger}^{\text {eff }}-\frac{E_{\mathrm{III}^{2}}}{A_{\mathrm{II}^{4}}}-\frac{Q}{A_{\mathrm{III}}{ }^{2}}=0,
$$

$$
\begin{gathered}
K_{\dagger}^{\mathrm{eff}} \equiv\left\langle\left[\int K(z)\right]^{2}\right\rangle\left[1+\frac{1}{24} \Phi\left(1+\frac{20}{27} c_{\mathrm{a}}\right)\right] ; \\
\epsilon_{\mathrm{III}}^{2} \equiv \epsilon^{2}\left[1+\Phi\left(1+\frac{1}{2} \Phi+3 \beta_{\mathrm{I}}\right)\right] .
\end{gathered}
$$

In Eq. (28), $c_{3}$ is of order unity (see Apps. $G, H$ ); in Eq. (29), $\beta_{1}$ is defined by Eq. (35). Roman-numeral subscripts on $A$ and $\in$ signify the order of approximationthird order in this cese. The subscript on $\beta \sim A^{-4}$ indicates that $A_{I}[\mathrm{Eq}$. (33)] is used to approximate $A$. The matching equation (27) is in the standard form of the smooth approximation, Eq. (33), and can be solved to find the third-order $A$ :

$$
A_{\mathrm{III}}^{2}=\frac{Q}{2 K_{f}^{\text {eff }}}+\left[\left(\frac{Q}{2 K_{f}^{\mathrm{eff}}}\right)^{2}+\frac{\epsilon_{\mathrm{III}}^{2}}{K_{\dagger}^{\text {eIf }}}\right]^{1 / 7} .
$$

If the input quantity is the mean radius $A_{\text {inp, then }}$ Eq. (27) gives the allowable $Q$ to third order,

$$
Q_{\text {III }}=A_{\text {inp }}^{2} K_{\dagger}^{\text {eff }}-\frac{E_{\text {III }}^{2}}{A_{\text {inp }}^{2}} \text {. }
$$

Second Order: Eq. (B 7 ) has two second-order terms. One yields the correction to $K^{\mathrm{eff}}$ seen in Eq. (28). The other term is $\alpha k^{2}\left\langle g^{2}\right\rangle$, or, using definition (18), $\alpha \Phi / 3$. We define

and get

$$
\epsilon_{\mathrm{II}}^{2} \equiv \epsilon^{2}(1+\Phi)
$$

$$
K_{\dagger}^{\mathrm{eT}}-\frac{E_{\Pi}^{2}}{A_{\mathrm{II}}{ }^{4}}-\frac{Q}{A_{\mathrm{II}^{2}}}=0 .
$$

Eq. (32), can be solved for $A_{\mathrm{II}}$ or $Q_{\mathrm{II}}$ in the same way as for the third order, giving useful approximations when $K(z)$ and $E$ produce $\sigma_{0}$ and $\sigma$ less than about $80^{\circ}$.

First Order: In Eq. (B7), the three terms of lowest order in $\alpha, q, h^{2}$ produce what is called the firgt-order matching equation in this paper (Ref. [9] used another terminology). This is the classic smooth approximation. These terms give $k^{2}\left\langle\left[\int h\right]^{2}\right\rangle=\alpha / 3+q$, or, using the definitions (14), (16), and (17),

$$
F^{\mathrm{eff}}-\frac{\epsilon^{\mathrm{I}}}{A_{\mathrm{I}}{ }^{4}}-\frac{Q}{A_{\mathrm{I}}{ }^{2}}=0 .
$$

The equations derived in this paper apply to arbitiary focusing profiles satisfying Eq. (3), such as the smooth profiles in Appendix G, but for illustration (Figs. 1, 2, and 3 ), we use the FODO model (Appendix $H$ ) with parameters from Table II. First-, second-, and third-order 
results for $A$, from (33), (32) and (30), are plotted in Fig. Ia for FODO. The smooth approximation is seen to be relatively inaccurate except near the point where its error curve crosses the $0 \%$ line.

\section{EXPLICIT THIRD-ORDER RESULT FOR}

Knowing the matched mean radius $A$, one can complete the solution for the beam envelope $a(z)=A(1+$ $\rho(z))$ using $\rho(z)$ from Eq. $(26) ; b(z)$ can be found by changing the sign of the terms that contain odd powers of $k$.

Some terms of Eq. (26) can be written in exact form [Appendix I] for models such as FODO, but Fourier expansion is more useful in general:

$$
h(z)=h_{1}\left[\cos \frac{\pi z}{L}+\frac{c z}{3} \cos \frac{3 \pi z}{L}+\frac{c_{5}}{5} \cos \frac{5 \pi z}{L}+\cdots\right] .
$$

Values (usually of order unity) of $h_{1}$ and $c_{n}$ for both smooth and FODO profiles are given in Appendices $G$ and H. With the definition

we have

$$
\beta_{1} \equiv \alpha_{1} \frac{L^{2}}{\pi^{2}}=3 \frac{L^{2}}{\pi^{2}} \frac{\epsilon^{2}}{A_{I}^{4}},
$$

$$
\begin{aligned}
a_{\mathrm{III}}^{\mathrm{max}}= & A_{I I I}\left[1+\rho_{\mathrm{II}}\left(1+\frac{c_{3}}{27}+\frac{c_{5}}{125}\right)+\right. \\
& \left.\frac{1}{8} \rho_{\mathrm{m}}^{2}\left(1+\frac{25 c_{3}}{54}\right)+\beta_{\mathrm{I}} \rho_{\mathrm{Im}}\left(1+\frac{5}{2} \rho_{\mathrm{m}}^{2}+\beta_{1}\right)\right]
\end{aligned}
$$

using results from Appendix E. The accuracy of Eq. (36) (applied to the special case of FODO model) is shown in Fig. 1b, along with that of the truncations

$$
a_{\mathrm{II}}^{\max }=A_{\mathrm{II}}\left[1+\rho_{\mathrm{m}}\left(1+\frac{1}{27} c_{3}+\frac{1}{125} c_{5}\right)+\beta_{\mathrm{I}} \rho_{\mathrm{m}}\right]
$$

and (the smooth approximation)

$$
a_{I}^{\max }=A_{I}\left(1+\rho_{\mathfrak{m}}\right) .
$$

The time dependence of Eq. (36), from App. E, is plotted in Fig. 2

\section{PHASE ADVANCES: ARBITRARY SYMMITRIC A-G WAVEFORMS}

From the well-lenown phase-amplitude result [13], the phase advance per quadrupole cell of length $2 L$ is

$$
\sigma=\epsilon \int_{0}^{2 L} \frac{d z}{a^{2}}=2 L \in\left\langle a^{-2}\right\rangle
$$

We approximate $a(z)$ by $A_{\text {III }}[1+\rho(z)]$ with $A_{\text {III }}$ from $\mathrm{Eq}$. (30) and $\rho(z)$ to third order from Eq. (26). Subscripts

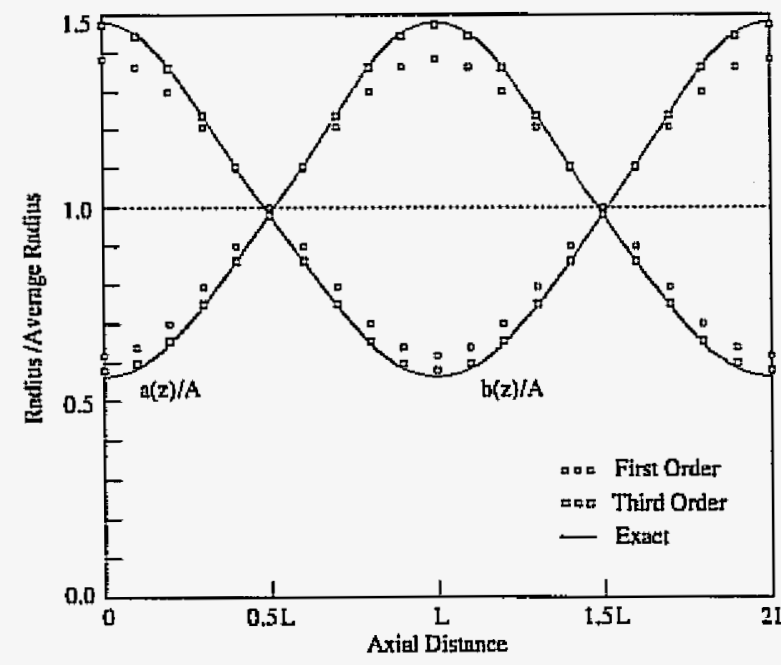

FIG. 2: Пllustration of matched envelopes $a(z)$ and $b(z)$ for special case of FODO model. Parameters from Table II, giving tunes $\sigma_{\mathfrak{n}}=112.2^{\circ}$ and $\sigma=86.9^{\circ}$. Exact envelopes (solid curves) were obtained numerfeally. Third-order results [Ege. (E6)-(E10)] give an $a_{\operatorname{mnx}}$ error of $-2.37 \%$. The smooth approximation error [Eq. (E6) only] is -13.0\%. Amplitude of half-period ripple is $5.6 \%$ of amplitude of full-period ripple.

are omitted for brevity. Expanding $a^{-2}$ and taling the average gives

$$
\sigma=2 L \frac{\epsilon}{A I^{2}}\left[1+3\left\langle\rho^{2}\right\rangle-4\left\langle\rho^{3}\right\rangle+5\left\langle\rho^{4}\right\rangle-\cdots\right] .
$$

(The $2 \rho$ term has zero average by definition.) Appendix $D$ shows that to third-order accuracy

$$
\sigma_{\mathrm{III}}=2 I_{\mathrm{I}} \frac{\epsilon}{A_{\Pi \mathrm{I}}^{2}}\left[1+\Phi\left(1+\frac{3}{4} \bar{\Phi}+2 \beta_{\mathrm{I}}\right)\right] \text {. }
$$

for arbitrery symmetric A-G waveforms. Errore with re spect to exact values from simulations are illustrated in Fig. 3a for the particular case of a FODO lattice. Usefull accuracy is retained after dropping two terms and using lower-order $A_{\text {II }}$ from Eq. (32):

$$
\sigma_{\mathrm{II}}=2 L \frac{\epsilon}{A_{\mathrm{II}}{ }^{2}}(1+\Phi) \text {. }
$$

Figure 3a shows large errors for the first-order result (smooth approximation):

$$
\sigma_{\mathrm{I}}=2 L \frac{\epsilon}{A_{1}^{2}} .
$$

The undepressed tume $\sigma_{0}$ for the general case is found by setting $Q=0$ in Eq. (27), then eliminating $\in$ from Eq. (40). Details are in Appendix D. The third-order result is

$$
\sigma_{0 \mathrm{III}}=2 L\left(K_{\dagger}^{\mathrm{eE}}\right)^{1 / 2}\left[1+\frac{1}{2} \Phi+\frac{7}{8} \Phi^{2}\right]
$$



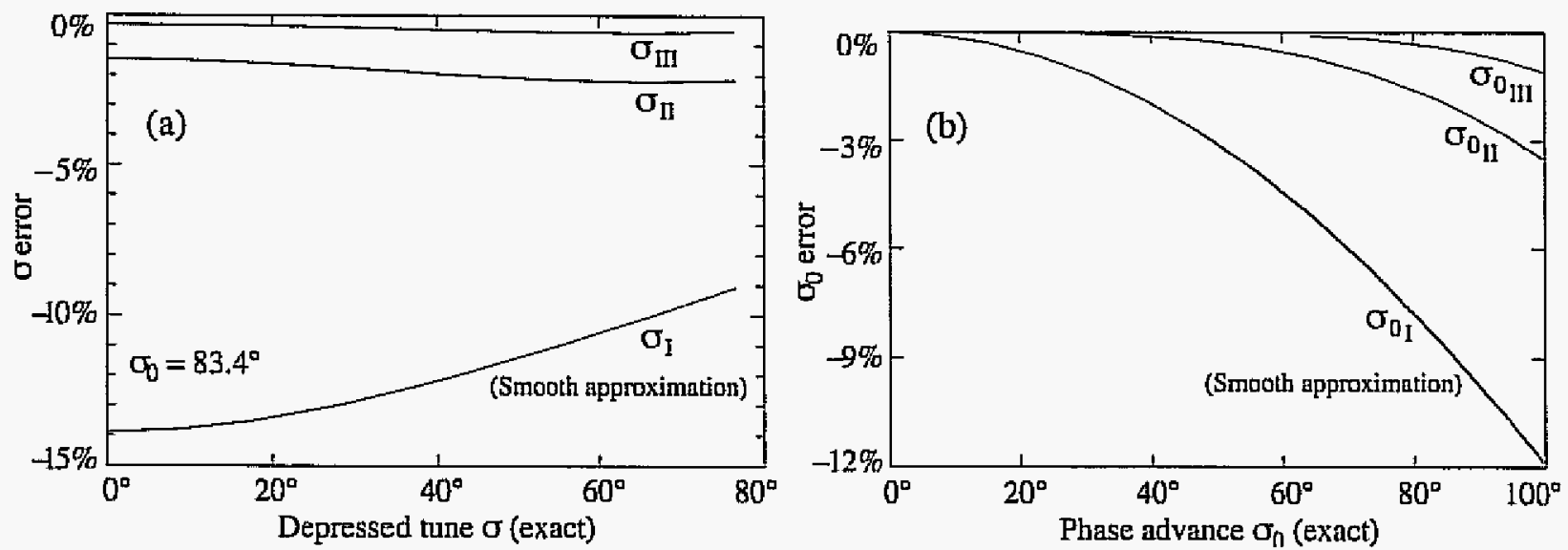

FIG. 3: Phase advances for special case of FODO model with parameters from Table II.

(a) Accuracy of depressed tume $\sigma$ from Eqs. (40)-(42). $V_{Q}$ is fixed at $20 \mathrm{kV}$ as in Fig. 1.

(b) Accuracy of phase advance $\sigma_{0}$ from Eqs. (43)-(45). $V_{Q}$ ranges from $5 \mathrm{kV}$ to $22 \mathrm{kV}$.

for arbitrary symmetric A-G waveforms. To secondorder,

$$
\sigma_{0 \mathrm{II}}=2 L\left(K_{\dagger}^{\mathrm{efr}}\right)^{1 / 2}\left[1+\frac{1}{2} \Phi\right] .
$$

The smooth approximation is

$$
\sigma_{0 \mathrm{I}}=2 L\left(K^{\mathrm{refF}}\right)^{1 / 2} .
$$

\section{UNDEPRESSED TUNE: SPECLAL CASE OF FODO WAVEFORM}

Figure 3 compares the accuracy of Eqs. (43), (44), and (45) for the special case of FODO focusing (with occupancy $\eta=0.5$ ), but gives an idea of the relative accuracy for arbitrary waveforms. At $\sigma_{0}=100^{\circ}$, the smooth approximation is off by $12 \%$ but this improves to $1 \%$ for third order.

For the FODO waveform, $\sigma_{0}$ is obtained exactly by multiplying transfer matrices [2]:

$$
\begin{aligned}
\sigma_{0}= & \cos ^{-1}\left[\cos \kappa \cosh \kappa+(1-\eta) K^{1 / 2} L(\cos \kappa \sinh \kappa\right. \\
& \left.-\sin \kappa \cosh \kappa)-\frac{1}{2}(1-\eta)^{2} K L^{2}(\sin \kappa \sinh \kappa)\right],
\end{aligned}
$$

where $\kappa=\eta K^{1 / 2} L$. Equation (46) applies to cases where $\sigma_{0}<180^{\circ}$. This exact solution can be expanded to give the close approximation

$$
\sigma_{0} \sim 2 \sin ^{-1}\left[L\left(K^{\mathrm{eff}}\right)^{1 / 2}\right]
$$

where for FODO, $K^{\text {eft }}=\frac{1}{12} \eta^{2}(3-2 \eta) k^{2} L^{2}$. Equation (47) resembles Eq. (45), the smooth approximation, but (for $\eta=0.5, \sigma_{0}=100^{\circ}$ ) the error is $0.41 \%$ instead of $12 \%$. The error increases with $\eta$, but is still only $1.1 \%$ for $\eta=1$.

The approximation (47) may be useful in non-FODO cases such as the examples in App. G; see App. L.

\section{APPENDTX A: DECOUPLING a AND b IN THE KV EQUATIONS}

This appendix calculates $a(z)+b(z)$ for the $Q$-term denominators in the KV equations. Dividing Eq. (1) by $A$ and expanding gives

$$
\begin{aligned}
\rho(z)^{\prime \prime}=-k h(z)- & k h(z) \rho+\frac{\epsilon^{2}}{A^{4}}(1-3 \rho+\cdots) \\
& +\frac{Q}{A^{2}}\left(1-R(z)+R^{2}(z)-\cdots\right)
\end{aligned}
$$

where

$$
R(z) \equiv \frac{\rho(z)+\rho(z+L)}{2} .
$$

We will find that only lowest order results are needed and drop the $R^{2}$ term, etc. We subtract the average of Eq. (A1), using definitions from Table I, and obtain

$$
\rho^{\prime \prime}=-k h(z)-k\{h \rho\}-\alpha \rho-q R(z)+\cdots,
$$

which we solve by iteration. In the initial step, the only known quantity is $l h h(z)$, so that

$$
\rho_{(0)}^{\prime \prime}=-k h, \quad \rho_{(0)}=-k g .
$$


Substituting $-\lg$ for $\rho$ in the previous equation,

$$
\begin{aligned}
& \rho_{(1)}^{\prime \prime}=-k h+k^{2}\{h g\}+\alpha h g ; \\
& \rho_{(I)}(z)=-h+k^{2} \delta+\alpha h \iint g,
\end{aligned}
$$

where we used the definition of $\delta$. Using the shift $\mathrm{Eq}$. (3c),

$$
\rho_{(1)}(z+L)=+k+k^{2} \tilde{d}-\alpha k \iint g \text {. }
$$

Then Eqs. (A2), (A6) and (A7) give

$$
R(z)=k^{2} \delta_{1}
$$

resulting in Eq. (1*) in Sec. III.

The expansion and iteration could be extended to produce more terms [all derived from $h(z)$ ] but these wotld give even smaller corrections to our results.

\section{APPENDEX B: DETAILS OF DERTVATION OF THE MATCHING EQUATION}

Rearranging Eq. (24) slightly gives

$$
h\langle h \rho\rangle=\alpha\left[\frac{1}{3}+2\left\langle\rho^{2}\right\rangle-\frac{10}{3}\left\langle\rho^{3}\right\rangle+5\left\langle\rho^{4}\right\rangle+\cdots\right]+q .
$$

Inserting $\rho$ from Eq. (26), the left-hand side of this equation is

$$
\begin{aligned}
k\langle h \rho\rangle & =k^{2}\left[-\langle h g\rangle+a\left\langle h \iint g\right\rangle+\frac{10}{3}-\alpha^{2} k^{2}\left\langle h \iint g^{3}\right\rangle\right. \\
& \left.-a^{2}\left\langle h \iiint \int g\right\rangle-k^{2}\left\langle h \iint h \delta\right\rangle-2 \alpha k^{2}\left\langle h \iint g \delta\right\rangle+\cdots\right]
\end{aligned}
$$

where we have dropped the subscript on $\rho$. The orthogonal $k^{2} \delta$ term is absent. We simplify by changing the order of integrations, using the $h(z)$ symmetries [Eq. (3)]. For example, $-\left\langle h \iiint \int g\right\rangle=-\left\langle g \iint g\right\rangle=+\left\langle\left[\int g\right]^{2}\right\rangle$. Applying this technique throughout gives

$$
\begin{aligned}
& k\langle h p\rangle=+k^{2}\left[\left\langle\left[\int h\right]^{2}\right\rangle+\alpha\left\langle g^{2}\right\rangle+\alpha^{2}\left\langle\left[\int g\right]^{2}\right\rangle\right]+ \\
& k^{4}\left[\left\langle\left[\int\{h g\}\right]^{2}\right\rangle+\frac{10}{3} \alpha\left\langle g^{4}\right\rangle+2 \alpha\left\langle g^{2} \delta\right\rangle+\cdots\right] .
\end{aligned}
$$

For the right-hand side of (B1),

$$
\begin{aligned}
& \left\langle\rho^{2}\right\rangle=k^{2}\left\langle g^{2}\right\rangle-2 \alpha k^{2}\left\{g \iint g\right\}+2 k^{4}\left\langle g \iint h \delta\right\rangle+\cdots \\
& \left\langle\rho^{3}\right\rangle=3 k^{4}\left\langle g^{2} \delta\right\rangle+\cdots \\
& \left\langle\rho^{4}\right\rangle=k^{4}\left\langle g^{4}\right\rangle+\cdots
\end{aligned}
$$

The very small $k^{4}\left\langle\delta^{2}\right\rangle$ term was omitted from $\left\langle p^{2}\right\rangle$. Again changing the order of integrations, the right-hand side of $(\mathrm{B} 1)$ becomes

$$
\begin{aligned}
\text { rhs } & =\alpha\left[\frac{1}{3}+k^{2}\left(2\left\langle g^{2}\right\rangle+4 \alpha\left\langle\left[\int g\right]^{2}\right\rangle\right)+\right. \\
& \left.k^{4}\left(4\left\langle g \iint h \delta\right\rangle-10\left\langle g^{2} \delta\right\rangle+5\left\langle g^{4}\right\rangle\right)\right]+q+\cdots .
\end{aligned}
$$

Four of the terms of (B6) combine with terms of (B2), so that

$$
\begin{gathered}
k^{2}\left\langle\left[\int h\right]^{2}\right\rangle+k^{4}\left\langle\left[\int\{h g]\right]^{2}\right\rangle= \\
\alpha\left[\frac{1}{3}+k^{2}\left(\left\langle g^{2}\right\rangle+3 \alpha k^{2}\left\langle\left[\int g\right]^{2}\right\rangle\right)+\right. \\
\left.k^{4}\left(4\left\langle g \iint h \delta\right\rangle-12\left\langle g^{2} \delta\right\rangle+\frac{5}{3}\left\langle g^{4}\right\rangle\right)+\cdots\right]+q,
\end{gathered}
$$

the matching equation from Eq. (B1). Each term (except $\alpha / 3$ and $q$ ) involves averages of functions of the focusing profile $h(z)$. Given any $h(z)$-obtained from a model such as FODO or measured on an actual quadrupole cell-these averages can be calculated once and for all, being constant coefficients of the terms in $a$ and $k$. Appendix $\mathrm{C}$ shows how to write $\mathrm{Eq}$. (B7) in simple form [Eq. (C9)].

\section{APPENDDX C: SIMPLIFICATION OF THE MATCHING EQUATION}

It is convenient to write the Fourier representation in the form

$$
h(z)=h_{1}\left[\cos \frac{\pi z}{L}+\frac{1}{3} c_{3} \cos 3 \frac{\pi z}{L}+\frac{1}{5} c_{5} \cos 5 \frac{\pi z}{L} \cdots\right] .
$$

The axial profile of the quadrupole gradient determines $h_{1}$ and $c_{n}$. Tables III and IV show that $h_{1}$ remains of the order of unity while $c_{3}$ and $c_{5}$ can change sign as the profile is varied. For the hard-edge quadrupole model (FODO) with occupancy $\eta=0.5$, Table IV shows

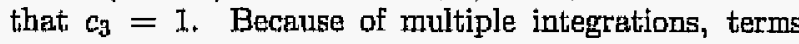
containing $c_{5}$ are usually negligible.

Rightl side of Eq. (B7): By definition, $k^{2}\left\langle g^{2}\right\rangle=\Phi / 3$. For the factor $\left\langle\left[\int g\right]^{2}\right\rangle=\left\langle\left[\iiint h\right]^{2}\right\rangle$, the third and higher harmonics make very small contributions because of the multiple integrations. Comparing leading terms for $\left\langle\left[\int g\right]^{2}\right\rangle$ and $\left\langle g^{2}\right\rangle$ gives

$$
3 k^{2}\left\langle\left[\int g\right]^{2}\right\rangle \approx \frac{L^{2}}{\pi^{2}} 3 k^{2}\left\langle g^{2}\right\rangle=\frac{L^{2}}{\pi^{2}} \Phi
$$


The three $k^{4}$ terms on the rhs of Eq. (B7) are:

$$
\begin{aligned}
4\left\langle g \iint h \delta\right\rangle & =\frac{1}{2}\left\langle g^{2}\right\rangle^{2}\left(1+\frac{19}{27} c_{g}+\cdots\right), \\
-12\left\langle g^{2} \delta\right\rangle & =-\frac{3}{2}\left\langle g^{2}\right\rangle^{2}\left(1+\frac{4}{9} c_{3}+\cdots\right), \\
\frac{5}{3}\left\langle g^{4}\right\rangle & =\frac{5}{2}\left\langle g^{2}\right\rangle^{2}\left(1+\frac{4}{81} c_{3}+\cdots\right) .
\end{aligned}
$$

Adding (C3) through (C5) grives $\frac{3}{2}\left\langle g^{2}\right\rangle^{2}(1+$ $\left.(29 / 243) c_{3}+\cdots\right)$, where the small $c_{3}$ correction can be neglected aince it corrects a term which is already third order. Using all these results along with definition (14), the right-hand side of (B7) (without the $q$ term) becomes

$$
\begin{aligned}
& \in^{2}\left(1+\Phi+\frac{1}{2} \Phi^{2}+\frac{9}{\pi^{2}} \frac{\epsilon^{2} L^{2}}{A_{I}{ }^{4}} \Phi\right)= \\
& \epsilon^{2}\left(1+\Phi+\frac{1}{2} \Phi^{2}+3 \beta_{I} \Phi\right) \equiv \epsilon_{I^{2}}{ }^{2},
\end{aligned}
$$

as in the main text. In the last term,

$$
\beta_{\mathrm{I}} \equiv 3 \frac{L^{2}}{\pi^{2}} \frac{\epsilon^{2}}{A_{\mathrm{I}}{ }^{4}}
$$

uses the lowest-order value for $A$ because this term is already of the highest order that we retain.

Left side of Eq. (BT): The $\left\langle\left[\left.\int\{h g\}\right|^{2}\right\rangle\right.$ term is

$$
\left\langle[\{h g\}]^{2}\right\rangle=\frac{1}{8}\left\langle g^{2}\right\rangle\left(1+\frac{20}{27} c_{g}+\frac{53}{36} c_{3}^{2}+\cdots\right)\left\langle\left[\int h\right]^{2}\right\rangle .
$$

Dropping the $c_{3}^{2}$ term in (C8) for simplicity, we define the LHS of (B7) as

$$
K_{\dagger}^{\mathrm{eff}}=k^{2}\left\langle\left[\mathrm{~J}^{2}\right]^{2}\right\rangle\left(1+\frac{1}{24} \Phi\left(1+\frac{20}{27} c_{3}\right)\right) .
$$

Altogether,

$$
K_{\dagger}^{\mathrm{EI}}=\frac{E_{\Pi \mathrm{II}}^{2}}{A_{\mathrm{III}}{ }^{4}}+\frac{Q}{A_{\mathrm{IIJ}}^{2}}
$$

which is Eq. (27).

\section{APPENDLX D: DEPRESSED AND UNDEPRESSED TUNES, GENERAL CASE}

Here we evaluate the expansion terms in Eq. (39), From Eqs. (B3-B5) in App. B,

$$
\begin{aligned}
3\left\langle\rho^{2}\right\rangle & =3 k^{2}\left\langle g^{2}\right\rangle+6 \alpha k^{2}\left\langle\left[\int g\right]^{2}\right\rangle+6 k^{4}\left\langle g \iint h \delta\right\rangle+\cdots, \\
-4\left\langle\rho^{3}\right\rangle & =-12 k^{4}\left\langle g^{2} \delta\right\rangle+\cdots, \\
5\left\langle\rho^{4}\right\rangle & =5 k^{4}\left\langle g^{4}\right\rangle+.
\end{aligned}
$$

From App. C, Eqs. (C2)-(C5),

$$
\begin{aligned}
6 \alpha k^{2}\left\langle\left[\int g\right]^{2}\right\rangle & \approx \frac{L^{2}}{\pi^{2}} 6 \alpha k^{2}\left\langle g^{2}\right\rangle=2 \beta_{I} \Phi_{1} \\
6 k^{4}\left\langle g \iint h \delta\right\rangle & =\frac{3}{4} k^{4}\left\langle g^{2}\right\rangle^{2}\left(1+\frac{19}{2 T} c_{3}+\cdots\right), \\
-12 k^{4}\left\langle g^{2} \delta\right\rangle & =-\frac{3}{2} k^{4}\left\langle g^{2}\right\rangle\left(1+\frac{4}{9} c_{3}+\cdots\right), \\
5 k^{4}\left\langle g^{4}\right\rangle & =\frac{15}{2} k^{4}\left\langle g^{2}\right\rangle^{2}\left(1+\frac{4}{81} c_{3}+\cdots\right) .
\end{aligned}
$$

When the last three are added, the $c_{3}$ coefficient is only 25/729. Dropping this and using the definitions gives to third-order accuracy

$$
\sigma=2 L \frac{\epsilon}{A_{\mathrm{II}}{ }^{2}}\left(1+\Phi \frac{3}{4} \bar{\Phi}^{2}+2 \beta_{1} \Phi\right) .
$$

Undepressed tune: Setting $Q=0,(27)$ is

$$
K_{\mathrm{T}}^{\mathrm{eff}}=\frac{\epsilon_{\mathrm{III}}^{2}}{A_{\mathrm{II}}{ }^{4}}=\frac{\epsilon^{2}}{A_{\mathrm{III}}{ }^{4}}\left(1+\Phi+\frac{1}{2} \Phi^{2}+3 \beta_{\mathrm{I}} \Phi\right)^{1 / 2} .
$$

The factor $E^{2} / A I^{4}$ in the last term can be replaced by $K^{\mathrm{eff}} \equiv h^{2}\left\langle[/ h]^{2}\right\rangle$, according to Eq. (33) with $Q=0$. Comparing with the definition of $\Phi$ and Fourier expanding as before, the last term, to lowest order, is $3 \Phi^{2}$ for $Q=0$. Thus, altogether

$$
K_{\dagger}^{\mathrm{EI}}=\frac{\epsilon^{2}}{A_{\mathrm{LI}}{ }^{4}}\left(1+\Phi+\frac{7}{2} \Phi^{2}+\cdots\right)^{1 / 2} \text {. }
$$

Malking a similar replacement in Eq. (40) for the case $Q=0$

$$
\sigma_{0}=2 L \frac{\epsilon}{A_{\amalg ! 1}}\left(1+\Phi+\frac{11}{2} \Phi^{2}+\cdots\right) .
$$

Using Eq. (D10) to eliminate $\in / A_{I I}{ }^{2}$,

$$
\begin{aligned}
\sigma_{0}=2 L\left(K_{\dagger}^{\mathrm{eff}}\right)^{1 / 2}(1 & \left.+\Phi+\frac{11}{2} \Phi^{2}+\cdots\right) \\
& \times\left(1+\Phi+\frac{7}{2} \Phi^{2}+\cdots\right)^{1 / 2}
\end{aligned}
$$

or, finally, to third order.

$$
\sigma_{0}=2 L\left(K_{\dagger}^{\mathrm{eff}}\right)^{1 / 2}\left(1+\frac{1}{2} \Phi+\frac{7}{8} \Phi^{2}+\cdots\right)
$$

for arbitrary symmetric A-G profiles. Sometimes it is convenient to work with the squares of $\sigma_{0}$ and $\sigma$, which are for third order

$$
\sigma_{0}^{2}=4 L^{2} K_{\dagger}^{\mathrm{efr}}\left(1+\Phi+2 \Phi^{2}\right)
$$

and

$$
\sigma^{2}=4 L^{2} \frac{\epsilon^{2}}{A_{\mathrm{III}}}\left(1+2 \Phi\left(1+\frac{5}{4} \Phi^{2}+2 \beta_{\mathrm{I}}\right)\right) .
$$


APPENDIX E: CALCULATION OF $a(z)$ AND $b(z)$ FOR GENERAL CASE

Using Fourier expansion, written as in Appendix C, $k h(z)=k h_{I}\left[\cos \frac{\pi z}{L}+\frac{1}{3} c_{3} \cos 3 \frac{\pi z}{L}+\frac{1}{5} c_{5} \cos 5 \frac{\pi z}{L} \cdots\right]$, and recalling $\rho_{m} \equiv h_{1} h L^{2} / \pi^{2}$, the terms of Eq. (26) are

$$
\begin{gathered}
-k g(z)=-\iint h=\rho_{\mathrm{m}}\left[\cos \frac{\pi z}{L}+\frac{1}{27} c_{3} \cos 3 \frac{\pi z}{L}\right. \\
\left.+\frac{1}{125} c_{5} \cos 5 \frac{\pi z}{L}+\cdots\right] \\
\alpha k \iint g=\beta \rho_{\mathrm{m}}\left[\cos \frac{\pi z}{L}+\frac{1}{243} c_{3} \cos 3 \frac{\pi z}{L}+\cdots\right] \\
k^{2} \delta(z)=k^{2} \iint\{h g\}=\frac{1}{8} \rho_{\mathrm{m}}^{2}\left[\left(1+\frac{10}{27} c_{3} \cdots\right) \cos 2 \frac{\pi z}{L}\right. \\
\left.\quad+\frac{5}{54} c_{3} \cos 4 \frac{\pi z}{L}+\cdots\right] \\
\frac{10}{3} \alpha k^{3} \iint g^{3}=\frac{5}{2} \beta \rho_{\mathrm{m}}^{3}\left[\left(1+\frac{1}{27} c_{3}+\cdots\right) \cos 2 \frac{\pi z}{L}\right. \\
\left.+\frac{1}{27}\left(\frac{2}{9} c_{3}+\cdots\right) \cos 3 \frac{\pi z}{L}+\cdots\right] \\
-\alpha^{2} k \iiint g=\beta^{2} \rho_{\mathrm{m}} \cos \frac{\pi z}{L}+\cdots
\end{gathered}
$$

The small final two terms from Eq. (26) have been omitted here for simplicity.

\section{The Significant Terms}

We drop small quantities in the above equations. The criterion is that they contribute less than two parts per thousand to the final result for a bad-case scenario: large focusing voltage (giving phase advance of $112^{\circ}$ ) and large $\beta$. This leaves

$$
\begin{gathered}
-k g=\rho_{\mathrm{m}}\left[\cos \frac{\pi z}{L}+\frac{1}{27} c_{3} \cos 3 \frac{\pi z}{L}+\frac{1}{125} c_{5} \cos 5 \frac{\pi z}{L} \cdots\right] \\
\alpha k \iint g=\beta \rho_{\mathrm{m}} \cos \pi \frac{\pi z}{L}+\cdots \\
k^{2} \delta(z)=\frac{1}{8} \rho_{\mathrm{m}}^{2}\left[\cos 2 \frac{\pi z}{L}+\frac{25}{54} c_{3} \cos 4 \frac{\pi z}{L}+\cdots\right] \\
\frac{10}{3} \alpha k^{3} \iint g^{3}=\frac{5}{2} \rho \rho_{\mathrm{m}}{ }^{3} \cos \frac{\pi z}{L}
\end{gathered}
$$

$$
-\alpha^{2} k \iiint \int g=\beta^{2} \rho_{\mathrm{m}} \cos \frac{\pi z}{L}+\cdots .
$$

Adding all these gives $\rho(z)$ to third-order accuracy. A few small terms were omitted as mentioned before. Setting $z=0$ gives $\rho_{\max }$ and $a_{\max }=A\left(1+\rho_{\max }\right)$ as presented in Section VII. Setting $z=L$ changes the sign of all terms, except the even term $k^{2} \delta(z)$, and yields $a_{\mathrm{m} n}$. $\alpha$ and $\beta$ are only needed to lowest order, i.e., $\alpha_{\mathrm{I}}$ and $\beta_{\mathrm{I}}$; we omit the subscripts here.

Results from Eqa. (E6)-(E10) are shown in Fig. 2 in the main text.

\section{APPENDLX F: FOURTER FEPRESENTATION, ARBITRARY SYMMETRIC CASES}

\section{Fourier Coefficients}

Recall from Section II that the focusing force $K(z)$ in the $\mathrm{KV}$ equations is written as

$$
K(z)=k h(z)
$$

with $h(0)=1$. Because of the symmetries [Eq. (3)] and initial conditions, there are only odd harmonics and no sine terms:

$$
h(z)=\sum_{1,3,5, \ldots} h_{\pi} \cos \frac{n \pi z}{L}
$$

with the condition

$$
\sum_{1,3,5, \ldots} h_{n}=1
$$

The Fourier coefficients are

$$
h_{n}=\frac{1}{L} \int_{0}^{2 L} h(z) \cos \frac{n \pi z}{L} d z .
$$

It is often convenient to define

$$
c_{n} \equiv n \frac{h_{n}}{h_{1}}
$$

where $c_{1}=1$ by definition and where $\left|c_{3}\right|$ usually turns out to be of order unity-see Tables III and IV. Then Eq. (F2) is written as

$$
h(z)=h_{1} \sum_{\mathbb{I}_{1} \mathfrak{3}_{1} \mathbf{5}_{1} \ldots+} \frac{1}{n} c_{n} \cos \frac{n \pi z}{L} .
$$

\section{Solution of Envelope Equation}

In the solution for $\rho(z)$, Eq. (26), the largest term is

$$
\rho_{(0)}(z)=-k g(z)=-k \int_{L / 2}^{z} d z^{\prime} \int_{0}^{z^{\prime}} h\left({ }^{\prime \prime}\right) d z^{\prime \prime},
$$

which with Eqs. (F2) and (F5) is

$$
-k g(z)=\frac{k L^{2}}{\pi^{2}} h_{1} \sum_{1,3,5, \ldots} \frac{c_{n}}{n^{3}} \cos \frac{\pi \pi x}{L} .
$$


The next largest term is

$$
\alpha k \iint g=\alpha \frac{k L^{4}}{\pi^{4}} h_{1} \sum_{1,3,5, \ldots} \frac{c_{n}}{n^{5}} \cos \frac{n \pi z}{L} .
$$

To achieve $1 \%$ accuracy, the first three series elements of (FB) are usually required, whereas for $\mathrm{Eq}$. (Fg), only the fundamenta] is needed [cf. Eq. (ER)].

The additional terms of Eq. (26), shown in Eqs. (E3) and (E4), are found with the help of trigonometric identities.

The mean square of the integral of $\mathrm{Eq}$. (F2) gives the effective force

$$
K^{\mathrm{eft}} \equiv k^{2}\left\langle\left[\int h\right]^{2}\right\rangle=h_{1}^{2} \frac{h^{2} L^{2}}{2 \pi^{2}} \sum_{1,3,5, \ldots} \frac{c_{n}^{2}}{n^{4}},
$$

which is used in the matching equation and for calculat ing undepressed phase advance.

The correction term $\Phi$ (used in evaluating phase advances, average radius or transportable current, etc.) is

$$
\Phi \equiv 3 k^{2}\left\langle g^{2}\right\rangle=3 h_{1}{ }^{2} \frac{k^{4} L^{4}}{2 \pi^{4}} \sum_{1,3,5, \ldots} \frac{c_{\pi}^{2}}{n^{6}} \longrightarrow 3 h_{1}^{2} \frac{k^{4} L^{4}}{2 \pi^{4}}
$$

since the harmonics contribute practically nothing. Dividing this into $\mathrm{Eq}$. (9), we find

$$
\Phi=3 K^{\mathrm{eff}} \frac{L^{2}}{\pi^{2}}\left(1+\frac{c_{3}^{2}}{81}+\cdots\right)
$$

which could be useful in certain calculations.

\section{APPENDIX G: SOLUTION FOR SOME SMOOTH LATTICE PROFILES}

All our results apply to an arbitrary symmetric focusing profile once its Fourier coefficients are known. Some simple but representative smooth models are discussed here. In each case, we use only the lowest required harmonics.

Field Model $1: h(z)=\cos \frac{\pi z}{L}$.

$$
h_{1}=1_{1} \quad c_{3}=0 \text {. }
$$

This is the model used by Lee [8].

Field Model 2: $K^{\prime \prime}(0)=0$.

$$
h_{1}=\frac{9}{8}, \quad c_{3}=-\frac{1}{3},
$$

with all the other coefficients zero except $c_{1}$, which is unity by definition. This choice gives a flat field at the midpoint of the quadrupoles, without the discontinuities
TABLE III: $h_{1}$ and $c_{n} \equiv n h_{n} / h_{1}$ and Tequiv for four smooth models.

\begin{tabular}{cccccccc}
\hline \hline Model & $h_{1}$ & $c_{1}$ & $c_{9}$ & $c_{5}$ & $c_{7}$ & $c_{9}$ & $T_{\text {dquiv }}$ \\
\hline$\# 1$ & 1 & 1 & - & - & - & - & 0.57 \\
$\# 2$ & $9 / 8$ & 1 & $-1 / 3$ & - & - & - & 0.69 \\
$\# 3$ & $3 / 4$ & 1 & 1 & - & - & - & 0.40 \\
$\# 4$ & $15 / 16$ & 1 & $1 / 2$ & $-1 / 2$ & - & - & 0.53 \\
\hline$\#$
\end{tabular}

of the hard-edge FODO model. From Eqs. (G1), (F9) and (F10) we get

$$
\begin{aligned}
K^{\mathrm{eff}} & =\frac{g^{2}}{8^{2}} \frac{k^{2} L^{2}}{2 \pi^{2}}\left(1+\frac{1}{9^{3}}\right), \\
\Phi & =3 \frac{9^{2}}{8^{2}} \frac{k^{4} L^{4}}{2 \pi^{4}}\left(1+\frac{1}{9^{4}}\right) .
\end{aligned}
$$

The third-harmonic correction for $\Phi$ can be neglected in most cases.

Field Model 3: $K^{\prime}(L / 2)=0$.

$$
h_{1}=\frac{3}{4}, \quad c_{3}=1 \text {. }
$$

This model is narrow, peaked at the quadrupole midpoints, with zero slope at the gap centers. It gives focusing strength equivalent to FODO [Eq. (I1)] having about $40 \%$ accupancy. The third-harmonic corrections to $K^{\text {eff }}$ and $\Phi$ are $1 / 81$ and $1 / 243$ respectively.

Field Model 4: $K^{\prime \prime}(0)=0$ and $K^{\prime}(L / 2)=0$.

$$
h_{\mathrm{I}}=\frac{15}{16}, \quad c_{3}=\frac{1}{2}, \quad c_{5}=-\frac{1}{2},
$$

which gives a fairly realistic profile (Fig. 4) and corresponds to FODO with $\eta \sim 53 \%$. The third- and fifthharmonic corrections are well under $1 \%$ for this case.

Table III summarizes the above results.

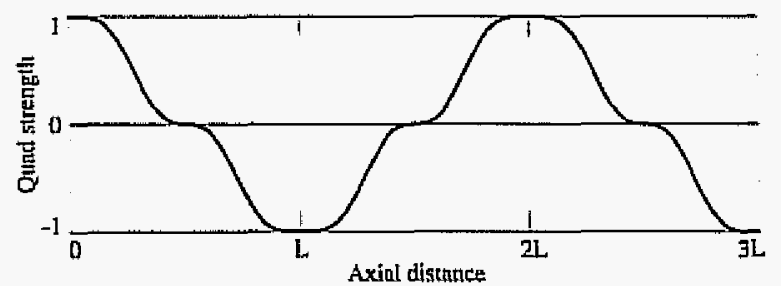

FIG. 4: Axial profile of normalized quadrupole strength $h(z)$ for Smooth Field Model $\frac{\|}{\pi} 4$, which tuses only the 3rd and 5th harmonics. The unit cell length is $2 L$. 
TABLE IV: $h_{1}$ and $c_{n} \equiv n h_{n} / h_{1}$ for FODO case with four different occupancies.

\begin{tabular}{cccccccc}
\hline \hline$\eta$ & $\pi h_{1} / 2$ & $c_{1}$ & $c_{3}$ & $c_{5}$ & $c_{T}$ & $c_{9}$ & $c_{11}$ \\
\hline $1 / 3$ & 1 & 1 & 2 & 1 & -1 & -2 & -1 \\
$1 / 2$ & $\sqrt{2}$ & 1 & 1 & -1 & -1 & 1 & 1 \\
$2 / 3$ & $\sqrt{3}$ & 1 & 0 & -1 & 1 & 0 & -1 \\
1 & 2 & 1 & -1 & 1 & -1 & 1 & -1 \\
\hline
\end{tabular}

\section{APPENDIX H: FOURTER SOLUTION FOR THE SPEGLAL CASE OF FODO}

In the case of the popular FODO lattice model (Fig. 5), the Fourier coefficients $h_{n}$ are readily calculated from Eq. (F4):

$$
h_{n}=\frac{4}{n \pi} \sin \frac{n \pi \eta}{2}
$$

which satisfies Eq. (F3) for occupancy $h$ over the range $0<\eta \leq 1$. From Eq. (F5),

$$
c_{n}=\pi \frac{\sin (n \pi \eta / 2)}{\sin (\pi \eta / 2)}
$$

for FODO. All the results from App. F can be used for FODO by putting $h_{1}=\sin \frac{\pi T_{1}}{2}$. Values of $h_{1}$ (normalized with $\pi / 2$ ) and $c_{n}$ are shown in Table IV for various $\eta$.

\section{APPENDTX I: SOME EXACT FORMULAS FOR THE SPECLAL CASE OF FODO}

Results using truncated Fourier representations for the hard-edge FODO may be compared with exact results by integration. (The FODO model is illustrated in Fig. 5.) Because of the symmetries expressed in Eq. (3), the $\mathrm{Cal}-$ culation of averages is simplified, requiring only integration over one-fourth of a cell. One finds for a specified

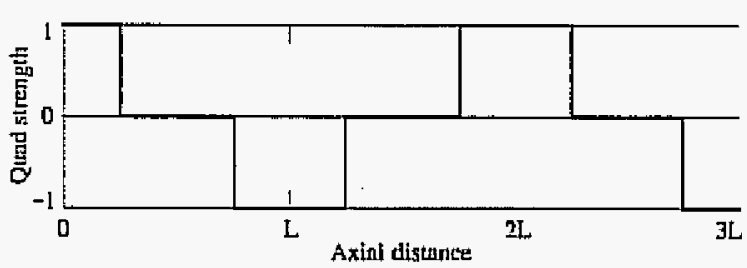

FIG. 5: Normalized quadrupole strength $h(z)$ vs. $z$ for a FODO lattice having occupancy 0.5 . The unit cell length is $2 L$. occupancy $\eta$

$$
\begin{gathered}
K^{\mathrm{eff}} \equiv k^{2}\left([j h]^{2}\right\rangle=\frac{1}{12} \eta^{2}(3-2 \eta) k^{2} L^{2} \\
\Phi \equiv 3 k^{2}\left(g^{2}\right\rangle=\frac{1}{16} \eta^{2}\left(1-\eta^{2}+\frac{2}{5} \eta^{3}\right) k^{2} L^{4} .
\end{gathered}
$$

One can do the integrals in the first two terms of $\rho(z)$, Eq. (26). For the integrations, we divide the cell into frve zones:

$$
\begin{array}{ll}
\text { Zone 0: } & 0 \leq z \leq \eta L / 2 \\
\text { Zone 1: } & \eta L / 2<z \leq L-\eta L / 2 \\
\text { Zone 2: } & L-\eta L / 2<z \leq L+\eta L / 2 \\
\text { Zone 3: } & L+\eta L / 2<z \leq 2 L-\eta L / 2 \\
\text { Zone 4: } & 2 L-\eta L / 2<z \leq 2 L .
\end{array}
$$

For even-numbered zones, the first integral is

$$
-\iint h=\mathbf{P}\left(\frac{\eta}{2}\right) \frac{1}{2}\left[\eta(2-\eta)\left(\frac{L}{2}\right)^{2}-\left(z-\pi \frac{L}{2}\right)^{2}\right]
$$

with $n$ the zone number. $P$ is defined as follows: for eny integer $m$,

$$
P(m) \equiv \begin{cases}+1 & \text { if } m \text { is even; } \\ -1 & \text { if } m \text { is odd. }\end{cases}
$$

For odd-numbered zones the integral is

$$
-\iint h=\mathbf{P}\left(\frac{n+1}{2}\right) \frac{1}{2} L_{\eta}\left(z-n \frac{L}{2}\right) .
$$

As required by the definition of $\iint$, the average has been subtracted. The maximum value of $\rho_{(0)}$ (where $z=0$, implying $n=0$ ) is

$$
\rho_{(0)}^{\max }=-\left.k \iiint h\right|_{0}=\frac{1}{8} \eta(2-\eta) k L^{2} .
$$

The next term in Eq. (26) includes the integral $\iint g$. For even-numbered zones

$$
\begin{aligned}
\iint g= & \mathbf{P}\left(\frac{n}{2}\right)\left[\eta\left(1-\frac{\eta^{2}}{2}+\frac{\eta^{3}}{8}\right)\left(\frac{L}{2}\right)^{4}-\right. \\
& \left.\frac{3}{4} \eta(2-\eta)\left(\frac{L}{2}\right)^{2}\left(z-\pi \frac{L}{2}\right)^{2}+\frac{1}{8}\left(z-\pi \frac{L}{2}\right)^{4}\right],
\end{aligned}
$$

and for odd-numbered zones

$$
\begin{aligned}
\iint g= & \mathbf{P}\left(\frac{n+1}{2}\right) \times \\
& {\left[\left(\frac{3}{2}-\frac{\eta^{2}}{2}\right)\left(\frac{L}{2}\right)^{3}\left(z-n \frac{L}{2}\right)-\frac{L}{4}\left(z-n \frac{L}{2}\right)^{3}\right] . }
\end{aligned}
$$

The maximum value of $\iint g$ is

$$
\left(\frac{L}{2}\right)^{4} \eta\left(1-\frac{\eta^{2}}{2}+\frac{\eta^{3}}{8}\right)
$$

These results, for any value of $\pi$, mey be compared with those from Appendices $G$ and $H$ to determine the number of Fourier terms needed for a given accuracy in each case. 


\section{APPENDIX J: NON-DIMENSIONAL FORMALISM}

As mentioned in Section $V$, we have chosen in this paper to use physical units for quantities such as the axial distance $z$. The result is that the expension parameters $k^{2}{ }_{1} q_{1}$ and $q$ are not necessarily small numbers. To show that they are nevertheless appropriate parameters, we replace $z$ by

$$
\theta \equiv \frac{\pi z}{L}
$$

The KV equations become

$$
\begin{aligned}
& \frac{\pi^{2}}{L^{2}} a(\theta)^{\prime \prime}=-K(\theta) a+\frac{\epsilon^{2}}{a^{3}}+\frac{2 Q}{a+b} \\
& \frac{\pi^{2}}{L^{2}} b(\theta)^{\prime \prime}=+K(\theta) b+\frac{\epsilon^{2}}{b^{3}}+\frac{2 Q}{a+b}
\end{aligned}
$$

where the primes now indicate differentiation with respect to $\theta$.

In Table I, averages are now defined by

$$
\langle f\rangle \equiv \frac{1}{2 \pi} \int_{0}^{2 \pi} f(\theta) d \theta .
$$

Similarly, the symbols $\int$ and $\iint$ refer to integration with respect to $\theta$, not $z$. Other replacements in Table I are

$$
\begin{aligned}
h(\theta) & \equiv \frac{K(\theta)}{K(0)}, \\
k & \equiv \frac{L^{2}}{\pi^{2}} K(0), \\
a & \equiv 3 \frac{L^{2}}{\pi^{2}} \frac{\in^{2}}{a^{4}}, \\
q & \equiv \frac{L^{2}}{\pi^{2}} \frac{Q}{A^{2}}, \\
K^{\mathrm{Eff}} & \equiv \frac{L^{2}}{\pi^{2}}(K(0))^{2}\left\langle\left[\int h\right]^{2}\right\rangle, \\
\rho_{\mathrm{m}} & \equiv h_{1} k .
\end{aligned}
$$

All these items are now dimensionless. In Eq. (15), the quantity $\beta$ is supplanted by $\alpha$. Equations (35) and (C7) no longer apply; $\beta$ must be replaced by $\alpha$ in Eqs. (29), (36), (37), (40), and (C6) as well as four places in App. D and six places in App. E. The equations throughout this paper are otherwise unchanged, and one can verify that the expansion parameters $k^{2}, \alpha$, and $q$ are now dimensionless small numbers.

\section{APPENDIX K: ALTERNATIVE MATCHING AND INITLAL CONDITIONS}

Another method of beam matching is convenient for some simulation studies. The beam is launched at the midpoint of a quadrupole gap. Using the same lattice models as before, the matching conditions would become

$$
\begin{aligned}
a(5 L / 2)=a(L / 2), & & b(5 L / 2)=b(L / 2), \\
a^{\prime}(5 L / 2)=a^{\prime}(L / 2), & & b^{\prime}(5 L / 2)=b(L / 2)
\end{aligned}
$$

with initial conditions

$$
a(L / 2)=b(L / 2), \quad b^{\prime}(L / 2)=-a^{\prime}(L / 2) .
$$

This choice would be essential for the case of unequal drift spaces (only a single symmetry) but we do not consider this case here.

\section{APPENDIX L: WORK FOR THE FUTURE}

Equation (47) is such a convenient approximation that it would be useful to find the types of A-G profiles for which it gives accurate results.

It would also be useful to investigate the modifications that would be required to extend the methods of this paper to non-symmetric A-G profiles.

\section{ACKNOWLEDGMENTS}

I thank E.P. Lee and S.M. Lund for helpful discussions. Special thanks to L.L. LoDestro for her contributions, including major improvements to Sections II and III. Supported in part by the U.S. Department of Energy under Contract DE-ACD3-76SF00098.
[1] I.M. Kapchinslej and V.V. Vladimirakij, Proc. Int. Conf. on High Energy Accel. and Instrum. p. 274 (GERN Sci. Inf. Service, Geneva, 1959).

[2] M. Reiser, Particle Accelerators 8, 167 (1978).

[3] J. Struclsmeier and M. Reiser, Particle Accelerators 14, $227(1984) ;$

[4] R.C. Davidson, Physics of Nonneutral Plasmas, AddisonWesley, New York, 1990.

R.C. Dayidson and Q. Qian, Phys. Plasmes $1_{1} 3104$ (1994).

[5] E.P. Lee, T.J. Fessenden, and L.J. Laslett, IEEE Trans. Nuc. Sci. NS-32, 2489 (1985).
[6] E.P. Lee, Particle Accelerators 52 (1996).

[7] O.A. Anderson, Lawrence Berkeley Laboratory report LBL-261233 (Revised), (1995).

[8] E.P. Lee, Phys. Plasmes 9, 4301 (2002).

[9] O.A. Anderson, Particle Accelerators 52, 133 (1996).

[10] O.A. Anderson, PAC 2005 Proceedings, p. 3535 (2005).

[11] S.M. Lund, S.H. Chilton, and E.P. Lee, Phys. Rev. ST Accel. Beame 9, 064201(2006).

[12] O.A. Anderson, et al., Nucl. Instrum. and Meth. $B 40 / 41,877$ (1989).

[13] E.D. Courant and H.S. Snyder, Ann. of Phys. 3, 1 (1958). 